\title{
ALTERACIONES NEUROPSICOLÓGICAS ASOCIADAS EN PACIENTES CON INFARTO LACUNAR
}

\author{
Luis I. Ledesma-Amaya ${ }^{*}$, Judith Salvador-Cruz ${ }^{1}$, Yaneth Rodríguez-Agudelo ${ }^{2}$, \\ Matilde Valencia-Flores ${ }^{3}$, Antonio Arauz ${ }^{2}$ \\ ${ }^{1}$ Facultad de Estudios Superiores - FES - Zaragoza, Universidad Nacional de Autónoma de México - UNAM, \\ ${ }_{2}^{2}$ Instituto Nacional de Neurología y Neurocirugía, Manuel Velasco Suárez - INNNMVS, \\ ${ }^{3}$ Facultad de Psicología, Universidad Nacional Autónoma de México - UNAM, Instituto Nacional de Ciencias Médicas \\ y Nutrición, Salvador Zubiran - INCMNSZ
}

Recibido, abril 25/2014

Concepto de evaluación, mayo 12/2014

Aceptado, mayo 28/2014
Referencia: Ledesma-Amaya, L.I., Salvador-Cruz, J., Rodríguez-Agudelo, Y., Valencia-Flores, M., Arauz, A. (2014). Alteraciones neuropsicológicas asociadas en pacientes con infarto lacunar. Acta Colombiana de Psicología, 17 (2), pp. 43-52. DOI:10.14718/ACP.2014.17.2.5

Resumen

Existe poca evidencia del deterioro cognoscitivo que defina el perfil del paciente con Infarto Lacunar (IL). El propósito de este estudio fue identificar las alteraciones neuropsicológicas en este tipo de pacientes. La muestra estuvo compuesta por 16 pacientes con IL y 16 participantes sanos con una edad promedio de $63 \pm 9.41$ y $64.75 \pm 9.06$ años y una escolaridad de $7.55 \pm 4.34$ y $7.94 \pm 3.51$, respectivamente. Se valoró la función cognoscitiva mediante un instrumento de tamizaje y una batería neuropsicológica. El análisis estadístico se llevó a cabo a través de la prueba Kruskal-Wallis y la U de Man-Whitney. Los pacientes con IL se caracterizan por la alteración de dominios como planeación, fluidez verbal, cambio atencional, habilidad visoconstructiva y velocidad de procesamiento de la información $(p<0.050)$. El IL se relaciona con el deterioro del funcionamiento ejecutivo y de velocidad de procesamiento, debido a la interrupción de circuitos frontocortico-subcorticales asociados con su correcto desempeño.

Palabras clave: Deterioro Cognoscitivo Vascular, cápsula interna, corona radiada, tallo encefálico, infarto cerebral, neuropsicología.

\section{NEUROPSYCHOLOGICAL DISORDERS ASSOCIATED WITH PATIENTS WITH LACUNAR INFARCT}

\begin{abstract}
There is little evidence of cognitive impairment to define the profile of patients with Lacunar Infarction (IL). The purpose of this study was to identify the neuropsychological disorders in these patients. The sample consisted of 16 patients with IL and 16 healthy control participants with an average age of $63 \pm 9.41$ and $64.75 \pm 9.06$ years, and average schooling of $7.55 \pm$ 4.34 and $7.94 \pm 3.51$, respectively. Cognitive function was assessed using a screening tool and a neuropsychological battery. Statistical analysis was carried out by the Kruskal-Wallis and Mann-Whitney U tests. Patients with IL are characterized by the alteration of domains such as planning, verbal fluency, attention switching, visoconstructive skill and information processing speed $(\mathrm{p}<0.050)$. The IL is related to the impairment of executive functioning and processing speed, due to the interruption of frontocortico-subcortical circuits associated with their proper performance.

Key words: Vascular Cognitive Impairment, internal capsule, corona radiata, brain stem, stroke, neuropsychology.
\end{abstract}

* Facultad de Estudios Superiores (FES) Zaragoza, Universidad Nacional de Autónoma de México (UNAM). Av. Guelatao \# 66, Col. Ejército del oriente, Deleg. Iztapalapa, Ciudad de México, C.P. 09230. Tel. 5255564470 77. luis_israel_ledesma@comunidad.unam.mx 


\title{
ALTERAÇÓES NEUROPSICOLÓGICAS ASSOCIADAS EM PACIENTES COM ENFARTE LACUNAR
}

\begin{abstract}
Resumo
Existe pouca evidência da deterioração cognoscitiva que defina o perfil do paciente com Enfarte Lacunar (IL). O propósito deste estudo foi identificar as alterações neuropsicológicas neste tipo de pacientes. A mostra foi composta por 16 pacientes com IL e 16 participantes sãos com uma idade média de $63 \pm 9.41$ e $64.75 \pm 9.06$ anos e uma escolaridade de $7.55 \pm 4.34$ e $7.94 \pm 3.51$, respectivamente. Avaliou-se a função cognoscitiva mediante um instrumento de tamizagem e uma bateria neuropsicológica. A análise estadística foi feita através do teste Kruskal-Wallis e a U de Man-Whitney. Os pacientes com IL se caracterizan pela alteração de dominios como planejamento, fluêcia verbal, mudança atencional, habilidade visoconstructiva e velocidade de processamento da informação $(p<0.050)$. O IL se relaciona com a deterioração do funcionamento executivo e da velocidade de processamento, devido à interrupção de circuitos frontocortico-subcorticais associados com seu correto desempenho.

Palavras chave: Deterioração Cognoscitiva Vascular, cápsula interna, coroa radiada, tronco encefálico, enfarte cerebral, neuropsicologia.
\end{abstract}

\section{INTRODUCCIÓN}

En México, la Enfermedad Vascular Cerebral (EVC) afecta al $0.2 \%$ de la población por año (Velásquez-Pérez, Juárez-Olivera, Jiménez-Marcial \& Trejo-Contreras, 2007). Los infartos subcorticales pequeños mejor conocidos como infartos lacunares (Jacova et al., 2012) comprenden el 25\% de todos los infartos cerebrales y son particularmente frecuentes en hispanos (Arauz, Murillo, Carlos, Barinagarramantiera, \& Higuera, 2003; Benavente et al., 2011). A pesar de la alta prevalencia del deterioro cognoscitivo y la demencia causados por un infarto lacunar, hasta el momento no existe una adecuada caracterización del deterioro relacionado con este trastorno (Jacova et al., 2012). Asimismo, Arboix (2011) y Filley y Brodtmann (2011) señalan que la relación de los síndromes motores y sensitivos con los infartos lacunares es ampliamente aceptada, pero aún no es claro el grado en el cual afectan la función cognoscitiva.

Los infartos lacunares se refieren a un infarto pequeño (de entre 0.2 a $15 \mathrm{~mm}$ ), profundo, atribuible a una enfermedad arterial primaria que envuelve a las ramas penetrantes de una arteria cerebral larga. Su localización es predominantemente subcortical, ganglios basales y tallo encefálico en áreas como putamen, tálamo, sustancia blanca de la cápsula interna, puente y ocasionalmente en la sustancia blanca del giro cerebral (Mohr et al., 2004). En general, la sintomatología que pueden presentar los pacientes que sufren de este trastorno está caracterizada por: un déficit neurológico de más de 72 horas de duración, cursando clínicamente en forma de hemiparesia motora pura, síndrome sensitivo puro, síndrome sensitivomotriz, hemiparesia atáxica y disartriamano torpe (Fisher, 1982).

Además de estos síndromes neurológicos, y dependiendo de su localización, los infartos lacunares pueden ocasionar un deterioro cognoscitivo y de la conducta (Bonelli \& Cummings, 2008). Este deterioro cognoscitivo relacionado con el infarto pertenece a la amplia gama de patología vascular que cubre el concepto propuesto por Hachinski \& Bowler (1993) llamado Deterioro Cognoscitivo Vascular (DCV). Al respecto, diferentes estudios neuropsicológicos coinciden en que existe un déficit cognoscitivo relacionado con el infarto lacunar.

Chen-Fu, Lan, Khor, Lai y Tai (2005), en una muestra de 23 pacientes con infarto lacunar, encontraron que $91 \%$ de ellos obtenían puntajes por debajo del punto de corte de la prueba CASI (Teng et al., 1994). De la misma manera, Fure, Bruun, Wyller, Engedal \& Thommessen (2006) estudiaron 71 pacientes con infarto lacunar agudo y describieron un déficit cognoscitivo presente en más del $50 \%$ de la muestra.

Por otro lado, Grau-Olivares, Arboix, Bartré \& Junqué (2007) estudiaron las alteraciones neuropsicológicas de 40 pacientes clasificados de acuerdo al síndrome lacunar (atípico, motor, sensitivo y disartria/mano torpe), encontrando que más del $50 \%$ de los pacientes presentaba Deterioro Cognoscitivo Vascular Leve (DCVL). Por último, Jacova et al. (2012), en un estudio de cohorte con 1636 pacientes con infarto lacunar sintomático, encontraron que el 50\% de la muestra cumplía con los criterios de DCVL con componente amnésico principalmente.

En cuanto a las alteraciones cognoscitivas específicas, Fure et al. (2006) observaron que pacientes con infarto lacunar agudo presentaban alteraciones principalmente de tipo ejecutivo, reducción de la velocidad psicomotora y déficit en memoria visual. Por otra parte, Grau Olivares et al. (2007) caracterizaron el infarto de acuerdo al tipo de síndrome y concluyeron que en el síndrome atípico se ven afectadas las funciones ejecutivas (fluidez verbal, categorial y fonética), la atención y las funciones premotoras. En el 
síndrome hemiparesis motora pura, se presentaron alteraciones en la comprensión del lenguaje y en las funciones premotoras; y finalmente, el síndrome sensitivo puro se asoció con un déficit concerniente al reconocimiento de la memoria visual.

En otro estudio, Geerlings, Appelman, Vincken, Mali \& Van der Graaf (2009) evaluaron a 522 pacientes con infarto lacunar, con el fin de investigar la asociación entre el volumen de las Lesiones en la Sustancia Blanca (LSB) y el número de infartos en relación con el funcionamiento de tipo ejecutivo y de memoria. Encontraron que no existe evidencia de una asociación del infarto lacunar con la alteración de la memoria; no obstante, observaron una disfunción ejecutiva en relación con el volumen de las LSB y el número de infartos.

Por otro lado, Baune, Roesler, Knech \& Berger (2009) evaluaron a 268 participantes con el fin de determinar la asociación entre infartos lacunares y LSB con distintas funciones cognoscitivas. Estos autores observaron que tanto las LSB como los infartos contribuyen en las alteraciones de diversos dominios cognoscitivos; específicamente, las LSB se relacionan con alteraciones en la memoria, la fluidez verbal, y de manera no clara con la velocidad de procesamiento de la información. En cuanto al infarto lacunar, se mencionó la existencia de una asociación con la velocidad de procesamiento y la función motora.

Por otra parte, Grau-Olivares et al. (2010) realizaron una investigación con 30 pacientes con infarto lacunar único, estudiando dos grupos, uno con DCVL y otro sin DCVL, con el objetivo de observar la progresión de la alteración cognoscitiva y los cambios cerebrales asociados. Los pacientes con DCVL presentaron mayor progresión (a los $18 \pm 6{ }_{\text {meses }} D E$ ) del déficit cognoscitivo global, y específicamente, en el deterioro de funciones como atención y memoria de trabajo; no obstante, observaron una mejoría en el reconocimiento de la memoria visual. Asimismo, encontraron pérdida del volumen de la sustancia gris regional en áreas corticales (frontal y temporal) y en regiones subcorticales (puente, cerebelo y caudado), debido a mecanismos de degeneración neuronal causados por lesiones vasculares cerebrales.

Recientemente, Jokinen et al. (2011), en un estudio longitudinal de tres años con 387 pacientes con infarto lacunar y LSB, determinaron que estos pacientes tenían un deterioro en dominios como velocidad de procesamiento cognoscitivo, atención selectiva y funcionamiento ejecutivo como control inhibitorio, cambio atencional y flexibilidad mental, independientemente del daño inicial, la progresión del padecimiento y el volumen de las LSB. Jacova et al. (2012) estudiaron a pacientes con infarto lacunar sintomático encontrando deficiencias en la función ejecutiva (en planeación y fluidez verbal) y destreza motora, resaltando la alteración de funciones como la velocidad de procesamiento y la memoria episódica.

Como se ha podido observar, el síndrome neuropsicológico asociado al infarto lacunar es heterogéneo y no existe una adecuada caracterización del mismo (Tripathi, Wang, Priyanka \& Spencer, 2011), ya que se habla de un déficit ejecutivo sin desprender o separar las principales características de este, y en asociación con otras alteraciones añadidas como la velocidad de procesamiento de la información. De la misma manera, hasta el momento no existe suficiente evidencia que aporte información al perfil cognoscitivo de este tipo de pacientes, principalmente provenientes de población latinoamericana. Por lo tanto, el propósito del presente trabajo es identificar las alteraciones neuropsicológicas de pacientes con infarto lacunar (Cápsula Interna/ Corona Radiada y Tallo encefálico), en comparación con un grupo de referencia en condiciones de salud normal.

\section{MÉTODO}

\section{Participantes}

Pacientes que asistieron a una institución de tercer nivel en la ciudad de México (Instituto Nacional de Neurología y Neurocirugía Manuel Velasco Suárez [INNNMVS]) diagnosticados con infarto lacunar en Cápsula Interna/ Corona Radiada (CI/CR) y Tallo Encefálico (TE) con uno a cinco años de evolución. La muestra se seleccionó de manera intencional, no probabilística. Se estudió un grupo de 16 pacientes (6 mujeres, 10 hombres) con infarto lacunar en $\mathrm{CI} / \mathrm{CR}$ y TE, en un rango de edad entre 50 y 80 años; todos los pacientes sabian leer y escribir. Se seleccionaron pacientes sin alteraciones de tipo neurológico diferente a la EVC, psiquiátrica y/o física, que impidiera la realización de pruebas neuropsicológicas; asimismo, se requería que dichos pacientes no presentaran deterioro cognoscitivo global (evaluado a través de la prueba CASI).

El grupo de comparación estuvo constituido por 16 participantes, 6 mujeres y 10 hombres, en condiciones de salud normal, con escolaridad y edad semejante al grupo de estudio y con un puntaje global del CASI de acuerdo al punto de corte de normalidad establecido por Lin et al. (2002) (escolaridad de 1-5 años 67/68 y de $6 \geq$ años 79/80).

\section{Instrumentos}

Las pruebas que se utilizaron fueron: el Instrumento de Screening de las Habilidades Cognoscitivas (CASI, por sus siglas en inglés) (Teng et al., 1994), como una prueba de tamizaje con el fin de evaluar el funcionamiento cognoscitivo global del paciente; pruebas para valorar funciones ejecutivas 
como planeación, a través del test de la Torre de Londres (Culbertson \& Zillmer, 1999), y el Test Ejecutivo del Reloj (Royall, Cordes, \& Polk, 1998); una sub-prueba de dígitos en inverso de la escala de inteligencia Weschsler para adultos (WAIS-III, Wechsler,1999), para valorar memoria de trabajo; el Test Controlado de Asociación Oral de Palabras (COWAT [PMR], Ross et al., 2007) asociado con la fluidez verbal; y el Test de los Cinco Dígitos (Sedo, 2007) para evaluar cambio atencional y control inhibitorio.

Adicionalmente, se utilizaron otras pruebas como el Test de Aprendizaje Verbal California (Elwood, 1995) para evaluar aprendizaje y memoria; una sub-prueba de dígitos en reverso de la escala de inteligencia Weschsler para adultos (WAIS-III, Wechsler, 1999) para valorar atención; la prueba de la Figura Compleja de Rey-Osterrieth (Rey, 1942) relacionada con habilidad visoconstructiva; y el Test de búsqueda de símbolos de WAIS III (Weschsler, 1999) para evaluar la velocidad de procesamiento de la información. Finalmente, también se aplicó el Inventario de depresión de Beck (Jurado et al., 1998), y el Inventario de apatía de Marín (Marin, Bidrzycki, \& Firinciogullari, 1991), con el fin de igualar los grupos de estudio y de referencia con respecto a su estado de ánimo y apatía.

\section{Procedimiento}

El infarto lacunar se estableció clínicamente por parte de un neurólogo vascular, basado en los criterios de clasificación de la Trial of Org in Acute Stroke Treatment (TOAST, por sus siglas en inglés) (Adams et al., 1993). El infarto lacunar se definió como un evento ocurrido en pacientes sin antecedentes de evento vascular. El historial clínico asociado al evento vascular fue confirmado por un neurólogo vascular a través de expedientes y estudios de neuroimagen por computadora de cada paciente. De la misma manera, el historial se valoró con respecto a enfermedades asociadas, o comorbilidades importantes para el estudio, así como información de antecedentes clínicos tales como factores de riesgo de infarto lacunar, diabetes, hipertensión arterial sistémica, consumo de sustancias, medicamentos empleados y datos de neuroimagen con diagnóstico radiológico de evolución, localización y número de infartos de cada uno de los pacientes (véase Tabla 1).

Para la evaluación neuropsicológica se solicitó a los pacientes y participantes la firma de un consentimiento informado que avalara su participación voluntaria. La evaluación neuropsicológica se realizó de manera individual con una duración aproximada de 2 horas, en un consultorio del departamento de neuropsicología del INNNMVS en condiciones óptimas

Tabla 1.

Descripción de los pacientes con infarto lacunar y grupo de referencia.

\begin{tabular}{|c|c|c|c|c|}
\hline Características demográficas & \multicolumn{3}{|c|}{$\begin{array}{c}\text { Grupo de estudio } \\
n=16\end{array}$} & $\begin{array}{c}\text { Grupo de referencia } \\
\qquad n=16\end{array}$ \\
\hline Hombres & \multicolumn{3}{|c|}{10} & 10 \\
\hline Mujeres & \multicolumn{3}{|c|}{6} & 6 \\
\hline Edad & \multicolumn{3}{|c|}{$63(9.41)$} & $64.75(9.06)$ \\
\hline Educación & \multicolumn{3}{|c|}{$7.13(4.03)$} & $7.94(3.51)$ \\
\hline \multicolumn{5}{|l|}{ Numero de infartos } \\
\hline Múltiple & \multicolumn{3}{|c|}{13} & \\
\hline Único & \multicolumn{3}{|c|}{3} & \\
\hline DM II & \multicolumn{3}{|c|}{2} & \\
\hline HAS & \multicolumn{3}{|c|}{7} & 1 \\
\hline DM II and HAS & \multicolumn{3}{|c|}{6} & \\
\hline Lado lesión & Derecha & Izquierda & $\%$ & \\
\hline Pons & 3 & 4 & $(43.8)$ & - \\
\hline Cápsula Interna & 3 & 2 & $(31.3)$ & - \\
\hline Corona radiada & & 2 & $(12.5)$ & - \\
\hline Mesencéfalo & 1 & & $(6.3)$ & - \\
\hline Corona radiata / centro semioval & & 1 & $(6.3)$ & - \\
\hline
\end{tabular}

Nota . DM II = Diabetes Mellitus tipo II, HAS = Hipertension Arterial Sistémica 
de iluminación y ventilación por dos especialistas en neuropsicología. El diseño de este estudio fue de tipo transversal.

\section{Análisis estadístico}

Las variables clínicas de los pacientes y sociodemográficas de los dos grupos de estudio se analizaron mediante estadística descriptiva e inferencial, a través de una prueba no paramétrica, elaborada en el programa computacional estadístico PASW® version 18 (una versión del programa SPSS). El procesamiento de los datos neuropsicológicos se realizó a través de la prueba U de Mann-Whitnney con el fin comparar los puntajes de las pruebas entre los grupos de estudio y referencia; asimismo, se utilizó la prueba de Kruskall-Wallis con el fin de comparar los dos subgrupos de infarto lacunar en CI/CR y TE con el grupo de referencia.

\section{RESULTADOS}

Se evaluaron 25 pacientes con diagnóstico de infarto lacunar, nueve de los cuales fueron excluidos debido a las siguientes situaciones: uno por déficit cognoscitivo severo, valorado a través de la prueba CASI (Teng et al,. 1994), un paciente con ceguera total, otro con una evaluación neuropsicológica incompleta, otro debido a causas ajenas a los investigadores, otro más con enfermedad neurológica diferente al infarto, y finalmente, cuatro con localización del infarto distinto a lo propuesto en este estudio.

Finalmente, el grupo de estudio estuvo compuesto por 16 pacientes (10 hombres, 6 mujeres) con una edad promedio de $M=63$ años, $D E=9.41$ y una escolaridad de $M=7.13$, $D E=4.03$, con diagnóstico de infarto lacunar único $(n=13)$ y múltiple $(n=3)$, con un período de evolución del infarto lacunar comprendido entre uno y cinco años. De estos 16 pacientes, ocho presentaron el infarto en la Cápsula Interna/ Corona Radiada y los ocho restantes en el Tallo Encefálico (principalmente en puente); nueve pacientes presentaron el infarto en el lado izquierdo y siete en el derecho. En cuanto al grupo de referencia, estuvo conformado por 16 participantes, (10 hombres, 6 mujeres) con una edad promedio de $M=64.75$ y $D E=9.06$ años y una escolaridad de $M=$ 7.94 y $D E=3.51$ años (véase Tabla 1 ).

En relación con los resultados neuropsicológicos, se observó que el grupo de estudio presentó un puntaje del desempeño cognoscitivo global bajo, en comparación con el grupo de referencia ( $p=.026$ ). Con respecto a funciones neuropsicológicas específicas, se encontró que el grupo de estudio presentaba alteración de dominios cognoscitivos como planeación $(p<.050)$, fluidez verbal PMR $(p<.050)$, cambio atencional $(p=.048)$, habilidad visconstructiva $(p=.001) \mathrm{y}$ velocidad de procesamiento de la información relacionada con la búsqueda visual $(p=.047)$ (Tabla 2).
Tabla 2.

Comparación de las pruebas neuropsicológicas entre grupos de pacientes y control

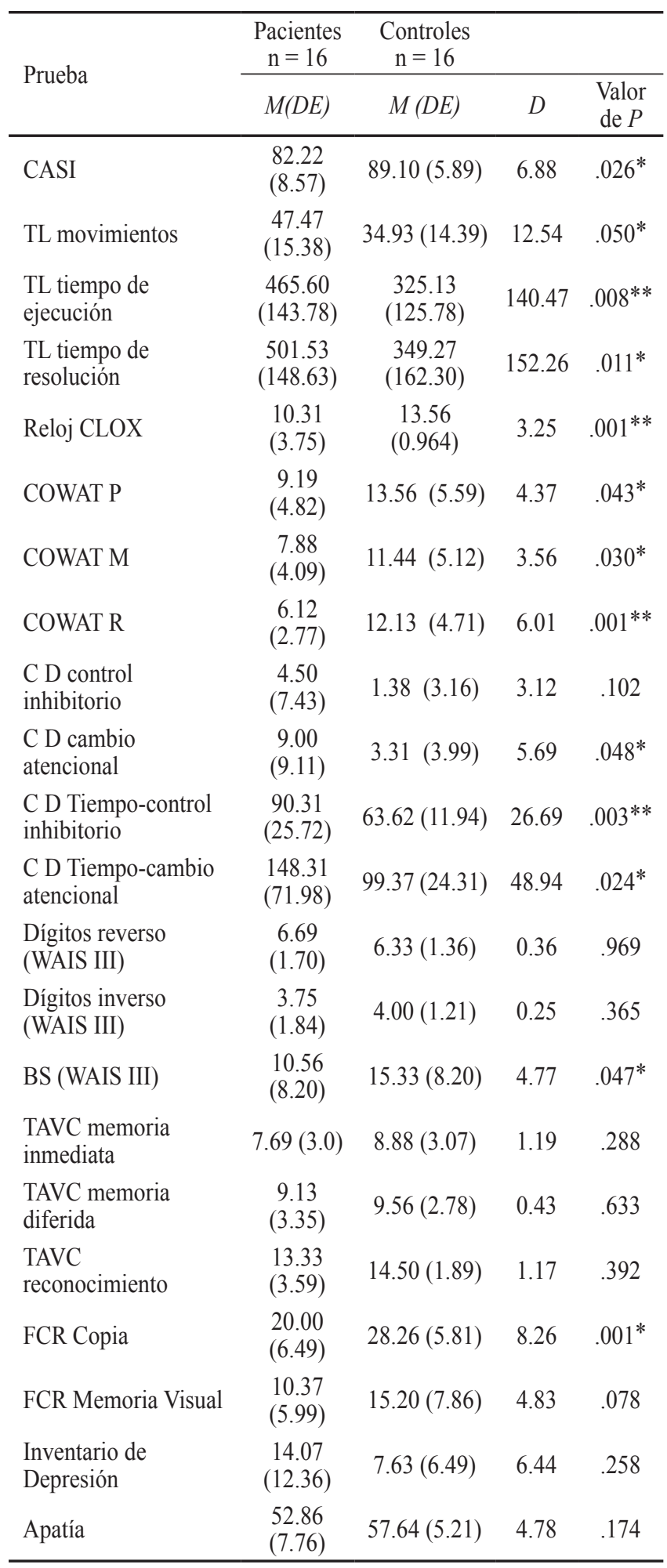

Nota. $\mathrm{D}=$ Diferencia, $\mathrm{TL}=$ Torre de Londres, $\mathrm{BS}=$ Búsqueda de símbolos, $\mathrm{CD}=$ Cinco Dígitos, $\mathrm{TAVC}=$ Test de Aprendizaje Verbal California, FCR $=$ Figura Compleja de Rey

*.05, ${ }^{* *} .001$ 
Tabla 3.

Comparación entre subgrupos de infarto lacunar en ganglios basales, tallo encefálico y grupo de referencia

\begin{tabular}{|c|c|c|c|c|c|c|}
\hline \multirow{3}{*}{ Prueba } & \multicolumn{2}{|c|}{ Localización del infarto } & \multirow{2}{*}{$\begin{array}{c}\text { G. Referencia } \\
n=16\end{array}$} & \multirow{3}{*}{$\begin{array}{c}\text { GR vs } \\
\text { GB y } \\
\text { TE } \\
\\
P\end{array}$} & \multirow{3}{*}{$\begin{array}{c}\text { GB vs } \\
\text { Control } \\
\\
P\end{array}$} & \multirow{3}{*}{$\begin{array}{c}\text { TE vs } \\
\text { Control } \\
P\end{array}$} \\
\hline & $\begin{array}{c}\mathrm{GB} \\
n=8\end{array}$ & $\begin{array}{c}\text { TE } \\
n=8\end{array}$ & & & & \\
\hline & $M(D E)$ & $M(D E)$ & $M(D E)$ & & & \\
\hline CASI & $83.45(9.47)$ & $81.48(8.42)$ & $89.10(5.89)$ & .078 & .158 & $.037^{*}$ \\
\hline Total de movimientos & $46(19.70)$ & $46.86(14.80)$ & $34.93(14.39)$ & .146 & .220 & .121 \\
\hline TL tiempo de ejecución & $462.75(160.65)$ & $468.86(134.55)$ & $349.27(162.30)$ & $.029^{*}$ & $.045^{*}$ & $.020^{*}$ \\
\hline TL tiempo de resolución del problema & $506.75(161.80)$ & $495.57(144.67)$ & $349.27(162.30)$ & $.038^{*}$ & $.039^{*}$ & $.038^{*}$ \\
\hline Reloj CLOX & $10.88(4.25)$ & $9.75(3.37)$ & $13.56(0.96)$ & $.004^{*}$ & $.025^{*}$ & $.002^{*}$ \\
\hline COWAT P & $8.38(3.46)$ & $7.88(4.83)$ & $13.56(5.59)$ & .081 & .256 & $.032^{*}$ \\
\hline COWAT M & $8.38(3.46)$ & $7.38(4.83)$ & $11.44(5.12)$ & .081 & .096 & .061 \\
\hline COWAT R & $5.00(1.30)$ & $7.50(3.33)$ & $12.13(4.71)$ & $.001^{*}$ & $.001^{* *}$ & $.029^{*}$ \\
\hline C D control inhibitorio & $1.50(3.46)$ & $7.00(9.57)$ & $1.38(3.16)$ & $.036^{*}$ & .947 & $.010^{*}$ \\
\hline C D alternancia atencional & $7.25(7.61)$ & $10.75(10.63)$ & $3.31(3.99)$ & $.085^{*}$ & .457 & $.014^{*}$ \\
\hline C D Tiempo-control inhibitorio & $88.12(29.85)$ & $92.50(22.70)$ & $63.62(11.95)$ & $.010^{*}$ & .061 & $.003^{*}$ \\
\hline C D Tiempo-alternancia atencional & $158.00(71.57)$ & $138.62(75.93)$ & $3.73(4.60)$ & $.046^{*}$ & $.016^{*}$ & .209 \\
\hline Dígitos reverso (WAIS III) & $6.75(1.58)$ & $6.63(1.92)$ & $6.33(1.36)$ & .909 & .754 & .801 \\
\hline Dígitos inverso (WAIS III) & $4.13(2.35)$ & $3.38(1.18)$ & $4.00(1.21)$ & .577 & .754 & .244 \\
\hline BS (WAIS III) & $11(8.48)$ & $10.12(8.47)$ & $15.33(8.20)$ & .131 & .164 & .065 \\
\hline TAVC memoria inmediata & $8.63(3.11)$ & $6.75(2.76)$ & $8.88(3.07)$ & .212 & .951 & .096 \\
\hline TAVC memoria diferida & $9.75(3.49)$ & $8.43(3.30)$ & $9.56(2.78)$ & .512 & .757 & .252 \\
\hline TAVC reconocimiento & $14.00(2.50)$ & $12.57(4.65)$ & $14.50(1.89)$ & .662 & .591 & .380 \\
\hline TAVC Aprendizaje & $9.00(2.61)$ & $10.88(2.53)$ & $10.75(3.83)$ & .662 & .780 & .116 \\
\hline FCR Copia & $20.50(7.56)$ & $19.50(5.71)$ & $28.26(5.81)$ & $.005^{*}$ & $.005^{*}$ & $.003^{*}$ \\
\hline FCR Memoria Visual & $10.687(6.54)$ & $10.062(5.81)$ & $15.20(7.86)$ & .212 & .137 & .165 \\
\hline Inventario de Depresión & $12.13(11.34)$ & $16.29(13.98)$ & $7.63(6.49)$ & .397 & .667 & .149 \\
\hline Apatía & $51.75(4.77)$ & $54.14(10.51)$ & $57.64(5.21)$ & .136 & .069 & .736 \\
\hline
\end{tabular}

Nota . $\mathrm{TL}=$ Torre de Londres, $\mathrm{BS}=$ Búsqueda de símbolos, $\mathrm{CD}=$ Cinco Dígitos, GR= Grupo de referencia, GB = Ganglios Basales, $\mathrm{TE}=$ Tallo encefálico

$* \mathrm{p}<0.05$

$* * \mathrm{p}<0.01$ 
$\mathrm{Al}$ realizar el análisis por subgrupos (CI/CR y TE) con el grupo control, se encontró que de manera específica la función ejecutiva en planeación, fluidez verbal con la letra $\mathrm{R}$ y el control inhibitorio $(p<.050)$ eran diferentes entre los tres grupos. Por otra parte, se observaron diferencias en la velocidad de procesamiento de la información de tareas como la ejecución y resolución de la Torre de Londres y cinco dígitos en tiempos de control inhibitorio y alternancia atencional $(p<.050)$. Finalmente, se presentaron diferencias significativas en la elaboración de la Figura Compleja de Rey (FCR), la cual valora habilidad viscontructiva ( $p=$ .005) (véase Tabla 3).

En relación con las comparaciones entre ambos subgrupos (CI/CR vs. TE), solo se observaron diferencias significativas en el desempeño de la fluidez verbal fonética (letra $\mathrm{R}, p=$ $.026)$ y el control inhibitorio $(p=.026)$. En la primera, el subgrupo de $\mathrm{CI} / \mathrm{CR}$ presentó un menor número de evocación de palabras $(M=5.00, \pm 1.309 D E)$ en comparación con el subgrupo TE $(7.50, \pm 3.338 D E)$; y en la segunda, el subgrupo de TE, exhibió un mayor número de errores relacionados con el control inhibitorio $(M=7.00, \pm 9.577$ $D E)$, a diferencia del subgrupo de $\mathrm{CI} / \mathrm{CR}(M=1.50 \pm 3.464$ $D E)$ (véase Tabla 3).

Finalmente, en cuanto al lado donde se ubicó el infarto, únicamente se observó que los pacientes con infarto lacunar izquierdo presentaban bajo desempeño cognoscitivo global $(p=.017)$ (CASI, $M=77.76, \pm 7.182 D E)$ en comparación con los pacientes cuyo infarto estaba ubicado en el lado derecho (CASI, $M=87.95, \pm 6.859 \mathrm{DE}$ ).

\section{DISCUSIÓN}

En este estudio se observó que pacientes con infarto lacunar presentan un desempeño cognoscitivo global bajo en comparación con los participantes sanos. De manera específica, se caracterizaron por la alteración de dominios que forman parte de la función ejecutiva como son la planeación, fluidez verbal y cambio atencional. Por otra parte, se observó un déficit existente en la habilidad visoconstructiva y la velocidad de procesamiento de la información, relacionada con tareas de tipo ejecutivo y de búsqueda visual, como son la resolución y ejecución de la prueba de planeación, alternancia atencional y control inhibitorio.

Con respecto a la alteración de la función ejecutiva, se explica probablemente por la interrupción de circuitos frontocortico-subcorticales que participan activamente en el correcto funcionamiento de este proceso cognoscitivo, ya que al ocurrir una lesión en dichos circuitos puede presentarse un bajo desempeño de este dominio (Bonelli \& Cummings, 2008; Cummings, 1993), el cual ha sido también descrito por otros autores como causa de un síndrome disejecutivo
(Junqué \& Mercé, 1990; Mungas, 2005) o sindrome frontalsubcortical (Young, 2011).

Congruente con los resultados observados en otros estudios (Baune et al., 2009; Grau-Olivares et al., 2007; Jacova et al., 2012; McMurtray, Liao, Haider, Lich \& Mendez, 2007; Reed et al., 2004) se encontró alteración de la fluidez verbal fonológica con las letras $P M R$, en relación con el grupo de estudio. Esta alteración se explica debido a que al paciente con IL, le demanda mayor tiempo y esfuerzo el procesamiento cognoscitivo para acceder al almacén lexical y encontrar las palabras compuestas por las letras que se le solicitan, y como consecuencia se produce una emisión menor de palabras observadas en la evaluación de la fluidez verbal.

Con respecto al deterioro de la velocidad de procesamiento de la información en estos pacientes señalamos que es un dominio cognoscitivo relevante en el correcto funcionamiento de tipo ejecutivo, el cual también está asociado con un circuito prefrontal-estriado encargado de proveer de velocidad a la información proveniente de zonas dorsolaterales del lóbulo prefrontal, teniendo como consecuencia una alteración de la función ejecutiva. Otra posible explicación se asocia con un estudio de neuroimagen en pacientes con lesiones lacunares relacionadas con la enfermedad llamada Arteriopatía autosomal dominante con infartos subcorticales y leucoencefalopatía (CADASIL, por sus siglas en inglés) en donde se observó que los pacientes presentaron un adelgazamiento cortical del lóbulo frontal medial izquierdo, paralelo a un enlentecimiento en la velocidad de procesamiento de la información, lo cual sugiere que las lesiones subcorticales pueden provocar un daño más extenso y relacionarse con una dismunición del volumen de zonas corticales que pueden propiciar un déficit cognoscitivo mayor, afectando principalmente la función ejecutiva (Duering et al., 2013).

Por otra parte, Salthouse (1985) señala que el enlentecimiento de la velocidad de procesamiento puede ser responsable de un declive en otras actividades cognoscitivas de orden superior. En el paciente con infarto lacunar, interviene en la alteración de tareas que están asociadas con distintos procesos ejecutivos. Tirapú-Ustárroz et al., (2011) mencionan que se desconoce hasta la fecha si este enlentecimiento en la velocidad de procesamiento afecta de igual modo a todos los procesos cognoscitivos o a unos de manera más intensa que a otros.

En cuanto a la alteración de la habilidad visoconstructiva, llama la atención que pocos estudios reportan una disfunción de este dominio en referencia a estos pacientes (McMurtray, et al. 2007; Chien-Fu, et al. 2005). Las elucidaciones que surgen con respecto a nuestro grupo de estudio y a esta alteración, se basan en que los pacientes 
presentaban una secuela de debilidad física no marcada pero observable por el evaluador, como consecuencia de un síndrome motor puro o sensitivo motor $(n=12$ pacientes $)$, lo cual afectó la ejecución de la copia del estímulo base de la figura compleja de Rey (FCR), provocando principalmente errores de distorsión del tipo precisión de la realización del trazo, cierre y tangencia en las unidades perceptuales. No obstante, se observó que estos pacientes presentaban un déficit en la planeación, es decir en la estrategia que siguen para la secuencia de elaboración del estímulo de la FCR.

En contraste con otros estudios (Benisty et al., 2009; Chen-Fu et al., 2005; Fure et al., 2006; Grau-Olivares et al., 2007), se observa la ausencia de deterioro de la memoria en este tipo de pacientes. Una posible explicación concierne a las características neuroanatómicas de la muestra utilizada, en comparación con otras de estudios antecedentes (Benisty et al. 2009; Reed, et al. 2004), fundamentalmente con respecto a localización del infarto en tálamo, o también conocido como infarto estratégicamente localizado, el cual ha sido reportado como responsable de un déficit cognoscitivo difuso y de memoria (Benisty et al., 2009; Kandiah et al., 2011; Reed, et al., 2004).

En cuanto a la comparación entre los subgrupos de CI/ $\mathrm{CR}$, TE y el grupo control, en relación con el funcionamiento cognoscitivo, se observó que los principales dominios alterados fueron la planeación, la fluidez verbal fonética (letra R), el control inhibitorio y el enlentecimiento en la velocidad de procesamiento de la información. Con respecto al análisis de comparación intragrupal, se pudo observar que pacientes con infarto en TE presentan una variación en el déficit de distintas funciones cognoscitivas de manera global, resaltando la velocidad de procesamiento, el control inhibitorio y el cambio atencional. La alteración de estas dos últimas, posiblemente se debe a la relación que guarda el sistema reticular activador ascendente, localizado en el tallo encefálico, el cual está asociado con mecanismos de alertamiento que son elementales para el funcionamiento del sistema atencional. Si ocurre una lesión en estas zonas de activación, encargadas de participar en el correcto flujo de la información que se envía al ejecutivo central localizado en el lóbulo prefrontal, se produce una disfunción en el control del sistema atencional, y por tanto, en funciones como el control inhibitorio y el cambio atencional.

Las aportaciones de este estudio se centran, en primer lugar, en la utilización de una muestra de origen latinoamericano, lo cual contribuye a brindar información sobre el perfil cognoscitivo del paciente con infarto lacunar de habla hispana, con características culturales y de escolaridad específicas, el mismo que ha sido estudiado recientemente por Benavente (2011) y Jacova (2012).
Por otra parte, la correcta selección de la muestra permitió descartar la presencia de demencia previa en nuestro grupo de estudio, hecho que de acuerdo con Edwards, Jacova, Sephery, Pratt, \& Benavente (2013) no se reporta en distintas exploraciones y se representa como una limitante en la definición del perfil de este tipo de pacientes.

Adicionalmente, el análisis neuropsicológico realizado en este estudio fue de tipo específico y se diferenció de otras investigaciones que se caracterizan por mencionar un deterioro de la función ejecutiva en relación con el infarto lacunar (Fure et al., 2006; Geerlings et al., 2009; GrauOlivares, 2007); no obstante, ninguna de esas investigaciones provee una información delimitada acerca de cuáles son aquellas funciones de tipo ejecutivo que se ven mayormente afectadas, circunstancia que por su especificidad beneficia al paciente en la adecuada elaboración e instauración de un plan de intervención.

En lo concerniente a las limitaciones de este estudio, cabe mencionar que el tamaño de la muestra fue una de ellas, ya que de haberse obtenido una muestra mayor se hubiera logrado un mayor poder estadístico en los resultados de la investigación. Por otro lado, una muestra representativa en relación con la variable número de infartos, hubiera permitido conocer las diferencias en el funcionamiento cognoscitivo entre pacientes con infarto lacunar único y múltiple. Asimismo, la clasificación del síndrome lacunar, como lo hecho en el estudio de Grau-Olivares et al. (2007), nos hubiera ofrecido información relevante en la comprensión del paciente con este padecimiento.

Finalmente, el seguimiento de este tipo de pacientes en un estudio longitudinal, propiciaría encontrar resultados asociados con la detección de cambios en el tiempo en dominios específicos del funcionamiento cognoscitivo del paciente con infarto lacunar.

En conclusión, y en concordancia con lo que señalan Arboix (2011) y Filley \& Brodtmann (2011), se acepta la representación clínica de los síndromes neurológicos motores y sensitivos en relación con el infarto lacunar, pero el grado en el cual los infartos afectan la función cognoscitiva no ha sido del todo descrita. Por consiguiente, el estudio del infarto lacunar, por su carácter sui generis, nos permite entender y asociar funciones cognoscitivas y territorios encefálicos con un importante aporte anatómico-funcional; asimismo, proporciona información a la actualización periódica que se hace con respecto a un concepto acuñado por Hachinski \& Bowler (1993) llamado Deterioro Cognoscitivo Vascular, del cual forman parte los infartos lacunares.

Como consecuencia, de manera prospectiva, la valoración y análisis neuropsicológico tiene repercusiones clínicas en la creación de programas de intervención enfocados en mejorar la calidad de vida de estos pacientes, como lo señalan Kolb 
y Whishaw (2008). El entendimiento de los mecanismos cognoscitivos en una valoración de lo que exactamente ha cambiado como resultado de la lesión cerebral debe ir precedida del diseño de los tratamientos de rehabilitación o de estimulación en dado caso, apoyando la valoración de aquellos que proporcionen validez social.

\section{REFERENCIAS}

Adams, H., Bendixen, B., Kappelle, L., Biller, J., Love, B., Gordon, D., \& Marsh, E. (1993). Classification of subtype of acute ischemic stroke. Definitions for use in a multicenter clinical trial. TOAST. Trial of Org 10172 in Acute Stroke Treatment. Stroke, 24, 35-41.

Arauz, A., Murillo, L., Carlos, C., Barinagarramantiera, F., \& Higuera, J. (2003). Prospective Study of Single and Multiple Lacunar Infarcts Using Magnetic Resonance Imaging. Stroke, 34, 2453-2458.

Arboix, A. (2011). Lacunar infarct and cognitive decline. Expert Review of Neurotherapeutics, 11(9), 1251-1252.

Baune, B., Roesler, A., Knech, S., \& Berger, K. (2009). Single and combined effects of cerebral white matter lesions and lacunar infaction on cognitive function in an erderly population. Journal of Gerontology: Medical Sciences, 64(1), 118-124.

Benavente, O., White, C., Pearce, L., Pergola, P., Roldan, A. B.-F., Christopher, C.,... Hart, R. (2011). The Secondary Prevention of Small Subcortical Strokes (SPS3) study. International Journal of Stroke, 6, 164-175.

Benisty, S., Gouw, A., Porcher, R., Madureira, S., Hernandez, K., Poggesi, A., . . Chabriat, H. (2009). Location of lacunar infarcts correlates with cognition in a sample of non-diabled subjects with age-related white-matter changes: the LADIS study. Journal of Neurology, Neurosurgery and Psichiatry, $80,478-483$.

Bonelli, R., \& Cummings, J. (2008). Frontal-Subcortical Dementias. The neurologist, 14(2), 100-107.

Chen-Fu, C., Lan, S., Khor, G; Lai, C \& Tai, C. (2005). Cognitive disfunction after acute lacunar infact. Kaohsiung Journal of Medical Sciences, 6(21), 267-271.

Culbertson, W., \& Zillmer, E. (1999). Tower of London Drexel University, examiner's manual resarch version. Toronto: Multi-Health Systems.

Cummings, J. (1993). Frontal-Subcortical Circuits and Human Behavior. Archives of neurology, 50, 873-880.

Duering, M., Gonik, M., Malik, R., Zieren, N., Reyes, S., Jouvent, E.,Dichgans, M. (2013). Identification of a strategic brain network underlyng processing speed deficits in vascular cognitive impairment. NeuroImage, 66, 177-183.

Elwood, R. (1995). The California Verbal Learning Test: Psychometric Characteristics and Clinical Application. Neuropsychology Review, 5(3), 173-201.
Filley, C. M., \& Brodtmann, A. (2011). Lacunes and cognitive decline. Neurology, 1856-1857.

Fisher, C. (1982). Lacunar strokes and infarcts: A review. Neurology, 32, 871-876.

Fure, B., Bruun Wyller, T., Engedal, K., \& Thommessen, B. (2006). Cognitive impairments in acute lacunar stroke. Acta Neurologica Scandinavica, 17-22.

Geerlings, M., Appelman, A., Vincken, K., Mali, W., \& van der Graaf, Y. (2009). Association of White Matter Lesions and Lacunar Infarcts With Executive Functioning. The SMART-MR Study. American Journal of Epidemiology, 170(9), 1147-1155.

Grau-Olivares, M., Arboix, A., Bartrés-Faz, D., \& Junqué, C. (2007). Neuropsychological abnormalities associated with lacunar infarction. Journal of neurological sciences (257), 160-166.

Grau-Olivares, M., Arboix, A., Junqué, C., Arenaza-Urquijo, E., Rovira, M., \& Bartrés-Faz, D. (2010). Progressive gray matter atrophy in lacunar patients with Vascular Mild Cognitive Impairment . Cerebrovascular Diseases, 157-166.

Hachinski, V., \& Bowler, J. (1993). Vascular Dementia. Neuro$\log y(43), 2159-2160$.

Jacova, C., Pearce, L., Costello, R., McClure, L., Holliday, S., Hart, R., \& Benavente, O. (2012). Cognitive Impairment in Lacunar Strokes The SPS3 Trial. 72(3), 351-362.

Jokinen, H., Gouw, A., Madureira, S., Yilikoski, R., Straaten, E., van der Flier, W., Erkenjuntti, T. (2011). Incident lacunes inflluence cognitive decline The LADIS study. Neurology, 76, 1872-1878.

Junqué, C., \& Mercé, J. (1990). Velocidad de procesamiento cognitivo en el envejecimiento. Anales de Psicología, 199-207.

Jurado, S., Villegas, E., Méndez, L., Rodríguez, F., Loperena, V., \& Varela, R. (1998). La estandarización del inventario de Depresión de Beck para los residentes de la ciudad de México. Salud Mental, 1(3), 26-31.

Kandiah, N., Wiryasaputra, L., Narasimhalu, K., Karandikar, A., Marmin, M., Chua, E., \& Sitoh, Y.-Y. (2011). Frontal subcortical ischemia is crucial for post stroke cognitive Impairment. Journal of Neurological Sciences, 92-95.

Kolb, B., \& Whishaw, I. (2008). Fundamentals of Human Neuropsychology. New York: Worth Publishers.

Lin, K.-N., Wang, P.-N., Liu, C.-Y., Chen, W.-T., Lee, Y.-C., \& Liu, H.-C. (2002). Cutoff Scores of the Cognitive Abilities Screening Instrument, Chinese Version in Screening of Dementia. Dementia and Geriatric Cognitive Disorders, 176-182.

Marin, R., Bidrzycki, R., \& Firinciogullari, S. (1991). Reability and validity of the Apathy Evaluation Scale. Psychiatry Research, 38, 143-162.

Mohr, J., Choi, D., Grotta, J., Weir, B., Wolf, P., \& Livingstone, C. (2004). Stroke, Pathophysiology, diagnosis and management. New York: Human Press. 
Mungas, D. (2005). Contributions of subcortical Lacunar Infarcts. En R. Paul, R. Cohen, B. Ott, \& S. Salloway, Vascular Dementia. Cerebrovascular Mechanisms and Clinical Management (pp. 211-222). New Jersey: Humana press.

McMurtray, A., Liao, A., Haider, J., Lich, E., \& Mendez, M. (2007). Cognitive performance after lacunar stroke correlates with leukoaraiosis severity. Cerebrovascular diseases, 24, 271-276.

Reed, B. E., Mungas, D., Weiner, M., Kramer, J., \& Jagust, W. (2004). Effects of white matter lesions and lacunes on cortical function. Archives of neurology, 61, 1545-1550.

Rey, A. (1942). L'examen psychologique dans les cas de encephalopathie traumatique. Archives de Psychologie,(28), 286-340.

Ross, T., Calhoun, E., Cox, T., \& Wenner, C. (2007). The reliability and validity of quialitative scores for the Controlled Oral Word Association Test. Archives of Clinical Neuropsychology, 22, 475-488.

Royall, D., Cordes, J., \& Polk, M. (1998). CLOX: An executive clock drawing task. Journal of Neurology, Neurosurgery and Psychiatry, 64, 588-594.

Salthouse, T. (1985). Speed of behavior and its implications for cognition. En J. Birren, \& K. Schale, Handbook of the psychology of aging (pp. 400-426). New York: Van Nostrand Reinhold.
Sedo, M. (2007). FDT-Test de los Cinco Digitos. Madrid: TEA ediciones.

Teng, E., Hasegwa, K., Homma, A., Imai, Y., Larson, E., Graves, A., Chiu, D. e. (1994). The Cognitive Abilities Screening Instrument (CASI): a practical test for cross-cultural epidemiological studies of dementia. International Psicogeriatric, 45-58.

Tirapú-Ustárroz, J., Luna-Lario, P., Hernáez-Goñi, P., \& García-Suescun, I. (2011). Relación entre la sustancia blanca y las funciones cognitivas. Revista de Neurologia, 52(12), 725-742.

Tripathi, R., Wang, K., Priyanka, M., \& Spencer, D. (2011). The influence of lacunes on cognitive function. Neurology, sup 111.

Velásquez-Pérez, L., Juárez-Olivera, S., Jiménez-Marcial, M., \& Trejo-Contreras, A. (2007). Epidemiología y tendencia del Evento Vascular Cerebral en el Instituto Nacional de Neurología y Neurocirugía Manuel Velasco Suaréz durante el período 1997-2003. Neurología, 22(1), 5-10.

Weschsler, D. (1999). WAIS III. Escala de inteligencia de Weschsler para adultos III. Manual Técnico. Madrid: TEA Ediciones.

Young, A. (2011). Vascular Dementia. Chonnam Medical Journal, 47, 66-71. 